\title{
Airway Compromise Due to Wound Hematoma Following Anterior Cervical Spine Surgery
}

\author{
Mark A. Palumbo ${ }^{*}$, , Jessica Pelow Aidlen ${ }^{1}$, Alan H. Daniels ${ }^{1}$, Nikhil A. Thakur ${ }^{1}$ and Joseph Caiati ${ }^{2}$ \\ ${ }^{I}$ Department of Orthopaedic Surgery and the ${ }^{2}$ Department of Anesthesia, The Warren Alpert Medical School of Brown \\ University, Providence, Rhode Island, USA
}

\begin{abstract}
One of the most serious adverse events associated with anterior cervical spine surgery is wound hematoma resulting in airway compromise. The reported incidence of this postoperative complication has varied from $0.2 \%$ to $1.9 \%$. Obstruction of the airway secondary to bleeding presents a challenging clinical scenario given the rapidity of onset, distorted anatomy of the upper respiratory tract, urgent need to act and potential for catastrophic consequences. This highrisk, life-threatening clinical scenario requires specialized knowledge and a well-designed treatment protocol to achieve a positive outcome. In this review, we report a case of airway compromise secondary to wound hematoma following anterior cervical discectomy and fusion, followed by a review of relevant literature, anatomy, etiologic factors and diagnostic considerations. We also propose guidelines for the prevention and management of postoperative airway obstruction due to wound hematoma.
\end{abstract}

Keywords: Anterior cervical spine surgery, adverse event, airway obstruction, hematoma.

\section{INTRODUCTION}

Anterior cervical spine operations are commonly performed by orthopedic and neurological surgeons. These procedures generally involve decompression of the neural elements by discectomy or corpectomy in combination with reconstruction of the spinal column at one or more levels. Available reconstructive techniques include arthrodesis (with or without instrumentation) and disc arthroplasty. Reported rates of clinical success are high and complications are generally infrequent.

One of the most serious adverse events associated with anterior cervical spine surgery is postoperative airway obstruction due to wound hematoma. The reported incidence of this complication has varied from $0.2 \%$ to $1.9 \%$ [1-4]. To date, there have not been any Level 1,2 , or 3 studies published examining the topic of airway compromise due to wound hematoma following anterior cervical surgery. Current treatment protocols are based on Level 4 and 5 data derived from case reports, retrospective series and expert opinion.

Airway compromise caused by hemorrhage presents a challenging clinical scenario given the rapidity of onset, limited time to act, and potential for catastrophic consequences including cerebral ischemia and death. Further, standard airway control measures may prove difficult to employ due to anatomic distortion of the upper respiratory tract and ventral neck.

Specialized knowledge and advance planning is necessary to respond efficiently and effectively in the setting

\footnotetext{
*Address correspondence to this author at the Division of Spine Surgery, Department of Orthopaedic Surgery, Alpert Medical School of Brown University, 2 Dudley Street, Suite 200, Providence, Rhode Island 02905, USA; Tel: 401-457-5195; Fax: 401-457-2141; E-mail: mpalmd@aol.com
}

of this life-threatening complication. Due to the low incidence of the problem, however, it is not universal policy for the medical team to formulate a specific protocol for the prevention and management of airway compromise. The primary objective of this review is to describe a systematic approach to the patient with airway obstruction due to wound hematoma after anterior cervical spine surgery. Guidelines for prevention and management will be preceded by a review of clinically relevant anatomy, etiologic factors and diagnostic considerations.

\section{CASE PRESENTATION}

A 52 year old female presented with a two year history of neck and right upper extremity pain along with paresthesias involving the thumb and index finger. The pain was progressive in nature and had become disabling despite comprehensive nonoperative management. Magnetic Resonance Imaging (MRI) showed a moderate paracentral disc herniation to the right of midline at the $\mathrm{C} 5 / 6$ level with compromise of the exiting C6 nerve root.

Her past medical history was significant for emphysema, bipolar disorder and epilepsy. There was no history of prior neck surgery. Physical examination was significant for low grade weakness of the right wrist extensor muscles and a decreased right brachialradialis reflex.

Anterior cervical discectomy and fusion (ACDF) was performed at the C5-6 level through a transverse, left-sided incision utilizing iliac crest autograft and anterior plate fixation (Fig. 1). There were no intraoperative complications. At the time of wound closure, hemostasis was adequate. The patient was extubated and transferred to the recovery room in stable condition. Six hours following completion of the operation, the patient began complaining of difficulty swallowing and breathing. She was agitated and tachypneic; oxygen saturation was $>95 \%$ on room air. Her neurologic exam was unchanged from her preoperative exam. 
Examination of her anterior neck revealed fullness and submandibular edema. The suture line was intact with no incisional drainage. The hemovac drain reservoir showed minimal bloody drainage and the tubing appeared to be clotted.

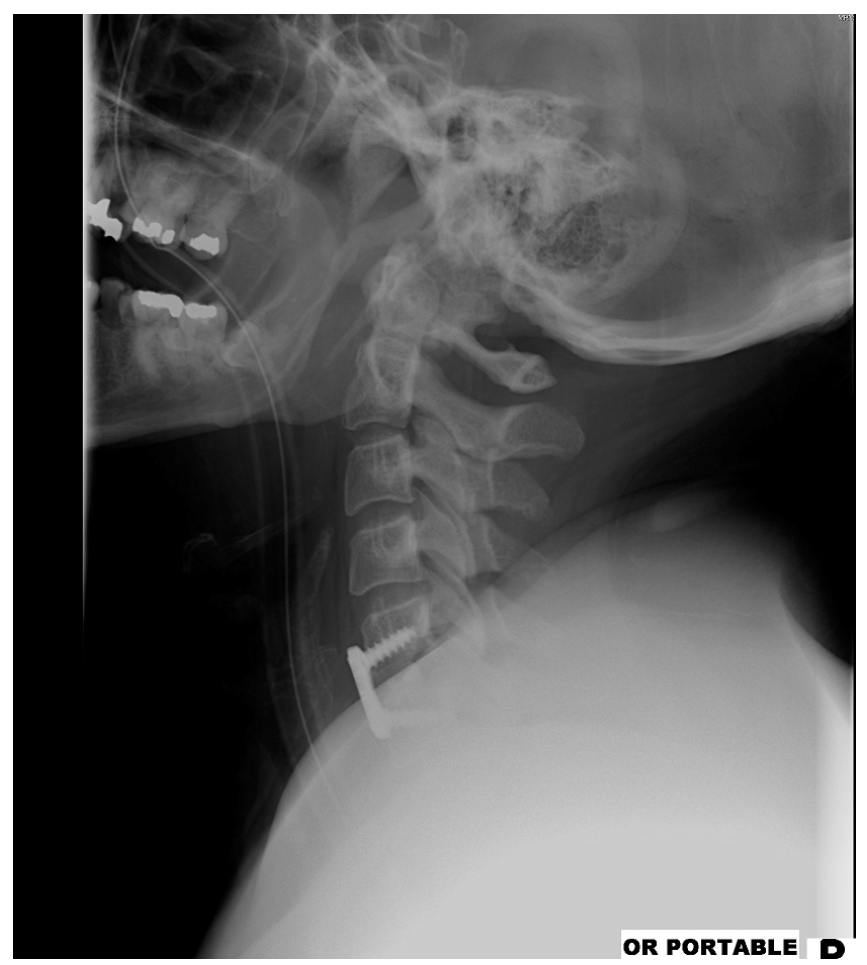

Fig. (1). Intraoperative lateral cervical spine xray showing anterior cervical discectomy and fusion of $\mathrm{C} 5 / 6$.

Immediate consultation with anesthesia and an otolaryngologist was obtained. The patient was taken emergently to the operating room. Bag-mask assisted ventilation was instituted. An attempt at awake intubation was compromised by poor visualization of the upper airway landmarks and edema of the pharyngeal wall, thus bag mask assisted ventilation was continued with the plan to attempt endotracheal intubation after hematoma evacuation. Next, the incision was opened under local anesthesia and a large hematoma was evacuated from the space between the carotid sheath and the midline viscera. After hematoma evacuation, standard endotracheal intubation was still unsuccessful due to upper airway edema. With the patient becoming more hypercarbic and tachycardic, emergent open tracheostomy was performed by the otolaryngologist through the prior leftsided incision, which was extended across the midline. After securing the airway, formal wound exploration revealed no clear source of arterial or venous bleeding.

Postoperatively, she was transferred to the intensive care unit. Her tracheostomy was removed on postoperative day \#4 and she was discharged home on the fifth postoperative day. The remainder of her postoperative course was unremarkable. She experienced major improvement in her preoperative pain symptoms and had normalization of strength in the right C6 innervated musculature.

\section{CLINICAL ANATOMY}

Effective management of airway compromise after cervical spine surgery requires an understanding of the surface anatomy of the anterior neck, the internal structure of the upper respiratory tract, and the surgical approach. The relevant external landmarks consist of the hyoid bone, the thyroid cartilage and the cricoid cartilage. Internally, the epiglottis separates the oropharynx from the laryngopharynx at approximately the level of the fourth cervical vertebra (Fig. 2). The larynx, extending from the lower pharynx to the trachea, is composed of a cartilaginous skeleton which includes the thyroid and cricoid cartilage along with the intervening cricothyroid membrane. Integration of ligaments and muscles with its cartilage components allows the larynx to serve as a soft tissue sphincter preventing passage of food and liquid into the tracheal lumen during swallowing.

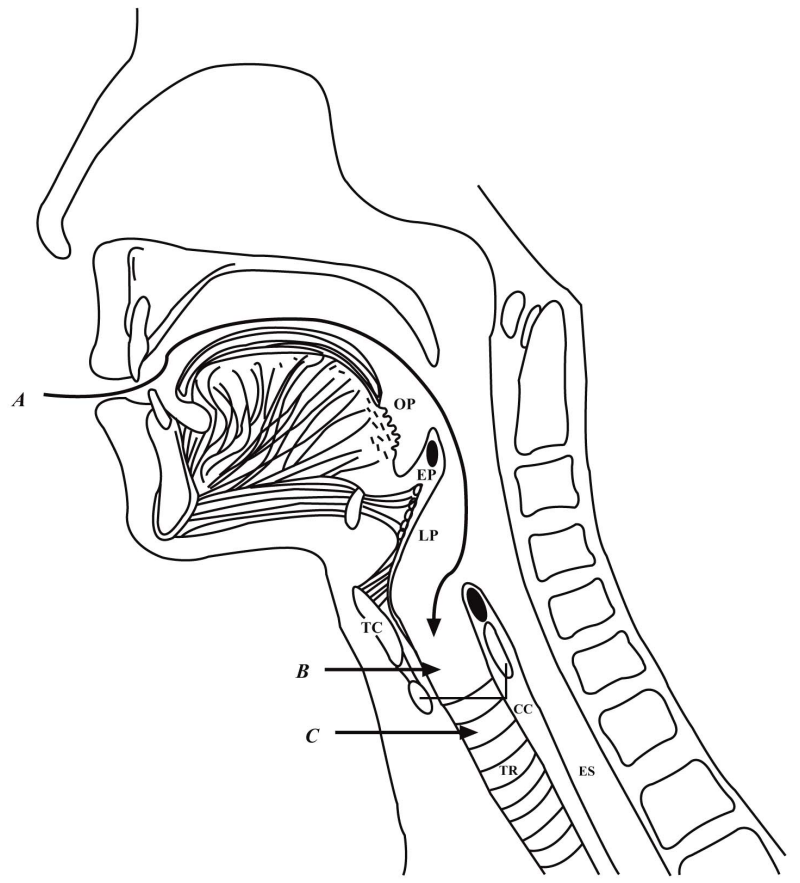

Fig. (2). Midsagittal section of the upper respiratory tract. OP: oropharnyx. LP: laryngopharnyx. TR: trachea. ES: esophagus. EP: epiglottis. TC: thyroid cartilage. CC: cricoid cartilage. Arrow $A$ : path for direct laryngoscopic orotracheal intubation. Arrow $B$ : path for surgical cricothyrotomy. Arrow $C$ : path for high surgical traceostomy.

The surgical exposure for anterior cervical spine surgery can be performed from either side of the midline via a transverse or longitudinal incision. Access to the vertebral column is achieved by dissection in the plane between the carotid sheath and the midline viscera (Fig. 3) [5]. During surgery, the upper airway structures and esophagus are retracted in a medial direction. The longus colli musculature is elevated off the ventral surface of the spine to expose the vertebral bodies and disc spaces. This exposure creates a potential space along one side and directly posterior to the larynx and trachea.

\section{ETIOLOGY}

Hematoma following anterior cervical spine surgery may be the result of inadequate control of arterial or venous bleeding during the operation, and has been reported due to superior thyroid artery dissection [6]. In other instances, a hematoma can form after surgery irrespective of adequate intraoperative hemostasis. Postoperative hemorrhage may 


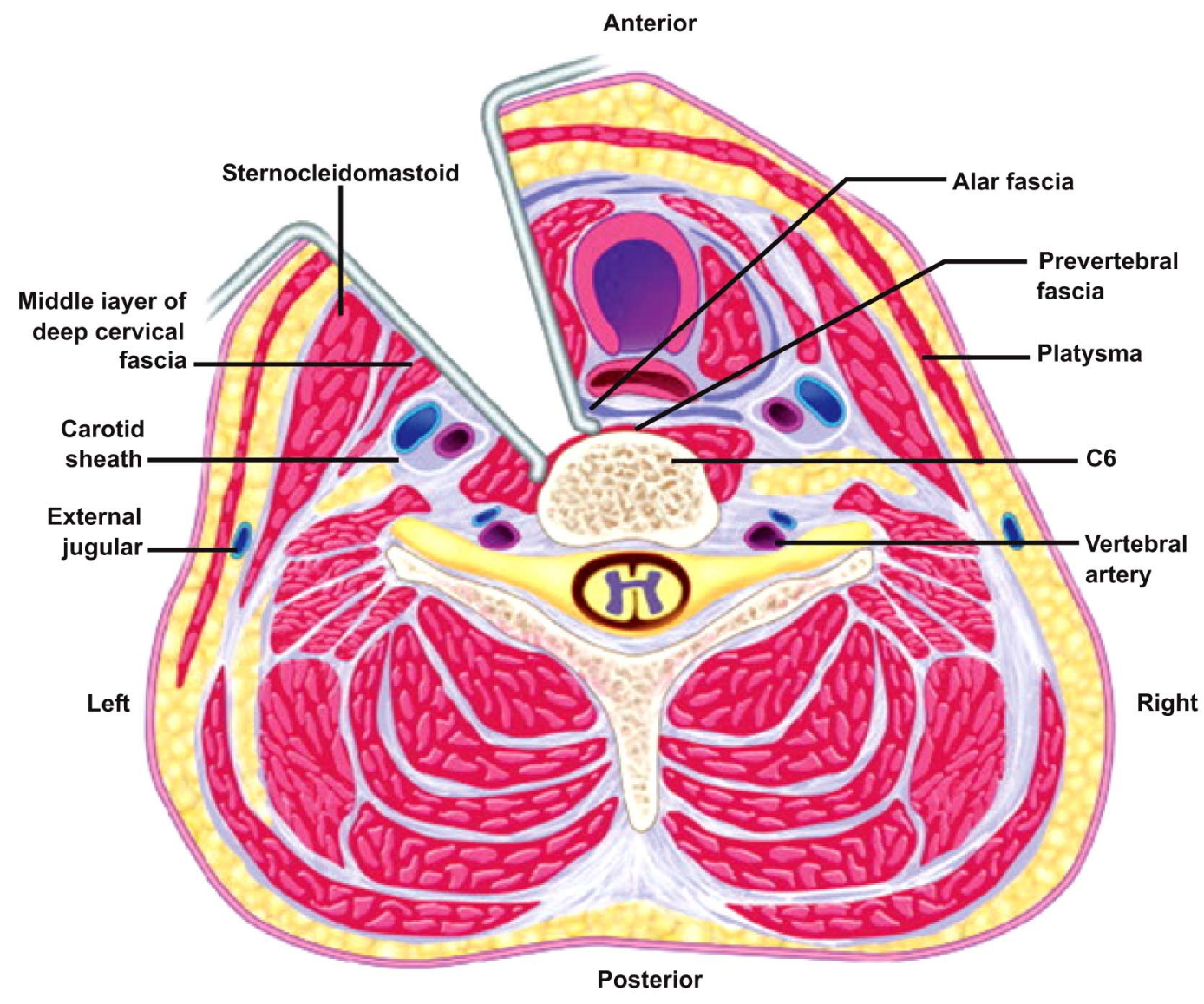

Fig. (3). The anterior cervical approach to the spine (cross-sectional view): note the potential space created by dissection in the plane between the carotid sheath and the midline viscera. The carotid sheath structures are retracted laterally, and the esophagus and trachea are retracted medially to expose the ventral surface of the spine. Adapted with permission from: Albert T, Balderston R, Northrup B. Surgical Approaches to the Spine. Philadelphia: WB Saunders 1997; p. 10 [5].

occur secondary to coagulopathy, increased blood pressure during emergence from anesthesia, or elevated venous pressure due to the Valsalva effect of coughing at the time of extubation [7].

There are two potential pathophysiologic mechanisms by which hematoma can produce airway compromise. The first is direct mechanical compression leading to reduction in the cross-sectional area of the airway lumen. The second mechanism involves the development of intrinsic airway edema in response to the mass effect of collected blood within the surgical wound. In this situation, impaired venous drainage from the head leads to secondary swelling of the supraglottic structures, the epiglottis and the arytenoids. This mechanism of progressive airway obstruction has been promoted as more plausible than extrinsic compression given the low pressure required to occlude the venous and lymphatic systems in the neck and the relatively high pressure required to directly collapse the airway [8].

Cephalad to and including the glottic opening (at the $\mathrm{C} 4$ level), the airway can be deformed and obscured by both external compression and internal edema of the layngopharyngeal soft tissue. Caudal to the glottic opening, the airway is more resistant to external compression due to support provided by the laryngeal and tracheal cartilage. The subglottic region, however, is vulnerable to luminal narrowing when mucosal edema develops within the confines of the cartilaginous skeleton. The individual or combined effects of edema and mechanical compression can compromise both spontaneous respiration and the execution of laryngoscopy.

\section{DIAGNOSTIC CONSIDERATIONS}

The underlying cause of airway compromise is suggested by the elapsed time to onset after the spine operation. Upper airway compromise that occurs during the immediate postoperative period (within 12 hours of completion of surgery) is most commonly the result of an expanding wound hematoma. It is noteworthy that obstruction evident within the first hour after extubation may be related to residual neuromuscular blockade or hypoventilation from narcotic medication. Although delayed hematoma is possible beyond the first twelve hours, alternative causes of airway obstruction (e.g. pharyngeal/prevertebral edema, spinal construct failure, cerebrospinal fluid collection or retropharyngeal abscess) become more probable.

In general, airway compromise after spine surgery can produce a spectrum of clinical findings. The patient may progress at a variable rate from being asymptomatic to exhibiting symptoms and signs of partial occlusion and then complete obstruction (Table 1). In the early stages, 
Table 1. Clinical Findings Associated with Postoperative Wound Hematoma

\begin{tabular}{|l|c|c|c|}
\cline { 2 - 4 } \multicolumn{1}{c|}{} & Respiratory & Neck & General \\
\cline { 2 - 4 } & Early & Elevated drain output & Restlessness \\
\cline { 2 - 4 } & Change in voice quality & Suture line bleeding & Agitation \\
\hline Late & Difficulty breathing & Anterior neck swelling & Panic \\
\hline Inspiratory stridor & Facial edema/plethora & Somnolence \\
\hline Cyanosis & Tracheal deviation & Unresponsiveness \\
\hline
\end{tabular}

complaints of difficulty talking and breathing predominate. Subtle changes in voice quality may be evident. Breathing problems may be exacerbated by the supine position. As the process evolves, the patient may become restless and agitated due to hypercarbia (with or without hypoxia). Dyspnea, inspiratory stridor and cyanosis develop during later stages of airway compromise with potential progression to respiratory failure/arrest.

Compared to other potential causative factors, wound hematoma tends to produce a more rapid rate of progression of respiratory compromise along with certain characteristic signs. Post-operative hemorrhage can be associated with significant drain output, suture line bleeding, progressive swelling of the anterior neck and submandibular region, facial edema/plethora and tracheal deviation [7, 9-11]. Sudden hemodynamic instability has been observed in the setting of an expanding neck hematoma after anterior cervical surgery. In the report by Sethi et al., bradycardia and hypotension was attributed to mechanical distortion of the carotid sinus with activation of the baroreceptor reflex [7].

\section{PREVENTION}

Wound hematoma after anterior cervical spine surgery is difficult to predict and prevent. The complication often occurs in the absence of predisposing factors. Nonetheless, the probability of postoperative hemorrhage can theoretically be reduced by attention to certain basic surgical principles. Specifically, an effort should be made to control all soft tissue and osseous bleeding during the operation. Placement of a closed suction drain is also reasonable (though with recognition that the measure will not negate the potential for hematoma formation).

Postoperative measures to protect the upper respiratory tract can be employed in the patient at elevated risk of hemorrhage into the wound. The presence of a bleeding diathesis (e.g. anticoagulant therapy, severe liver disease, hereditary bleeding disorder) and/or excessive intraoperative blood loss should raise concern for bleeding after surgery. Monitoring in an intensive care unit for 24-72 hours (with or without delayed removal of the endotracheal tube) should be considered. The threshold for delaying extubation should be even lower in the presence of additional risk factors for postoperative airway compromise [12-14] (Table 2).

\section{MANAGEMENT OF POSTOPERATIVE AIRWAY COMPROMISE}

Postoperative airway compromise due to hematoma poses a challenging clinical scenario. This situation typically involves a patient who is extubated immediately after surgery and then develops respiratory distress after transfer to the post-anesthesia care unit or the surgical ward. The time to onset of this complication ( $<12$ hours after surgery) is such that the on-site night staff will often have responsibility for acute management of the patient $[7,9,11]$.

An efficient and systematic approach to treatment is necessary to achieve a positive outcome. Early detection and intervention before the onset of occlusive laryngopharyngeal edema generally portends a positive outcome. The initial assessment functions to characterize the severity of airway obstruction. Based on this clinical information, the appropriate location to render care and the specific treatment methodology is established.

\section{INITIAL ASSESSMENT}

Following anterior cervical spine surgery, a patient with symptoms attributable to the airway must be evaluated immediately. If available in the hospital, the responsible spine surgeon and anesthesiologist should assess the situation. At this point, preparation to secure the airway is a priority; multispecialty assistance should be mobilized as rapidly as possible.

Upon reaching the bedside, the first responders must rapidly assess the clinical situation. A focused physical examination incorporates removal of the orthosis, visualizat-

Table 2. Additional Risk Factors For Postoperative Airway Compromise

\begin{tabular}{|c|c|c|c|}
\hline Surgical & Patient & Anesthetic & Institutional \\
\hline \hline Exposure of $>3$ vertebral bodies & Morbid Obesity & Suboptimal airway visualization & No 24 hour in-house anesthesia care \\
\cline { 1 - 2 } Exposure of C2-C4 levels & Obstructive Sleep Apnea & Multiple intubation attempts & \multirow{2}{*}{ Need for fiberoptic intubation } \\
\cline { 1 - 2 } Blood loss $>300 \mathrm{ml}$ & Pulmonary disease & No 24 hour in-house surgical staff \\
\cline { 1 - 2 } Operative time $>5$ hours & Cervical Myelopathy & \\
\hline
\end{tabular}


ion of the anterior neck tissues and identification of signs of airway obstruction. Identification of an expanding wound hematoma is based on exam findings and knowledge of the time elapsed since surgery. Any other immediately available data are taken into consideration (e.g. pulse oximetry, review of patient comorbidities, assessment of available hospital resources, etc).

The severity of airway compromise is characterized in order to determine whether the patient can be safely transported to the operating room. Non-critical airway compromise is associated with minimal/partial loss of airway patency. Clinically, the patient is not in extremis. Complaints of difficulty breathing and talking may be accompanied by dyspnea and subtle changes in voice quality. Characterization of the airway compromise as non-critical reflects a clinical judgment that the situation is not immediately life-threatening and that transfer of the patient to the operating room is reasonable.

Critical airway compromise is associated with near-total or completes loss of airway patency. Representative signs include a look of fear or panic associated with air hunger, excessive salivation, a rocking motion of the head and chest with the respiratory cycle, use of accessory muscles of respiration, inspiratory stridor and, eventually, central cyanosis. Characterization of the airway compromise as critical is indicative of a situation judged to be immediately life-threatening; management must be initiated at the bedside.

\section{GENERAL GUIDELINES}

The primary treatment objective is to establish and maintain patency of the airway by placement of an endotracheal tube. Intubation can be achieved by direct laryngoscopy, fiberoptic bronchoscopy, or by surgical means. In this regard, there are certain fundamental principles pertinent to the emergency management of airway compromise after anterior cervical spine surgery.

- The optimal setting to secure the airway is in the operating room suite.

$\bullet$

When supplemental oxygen is available, delivery of $100 \%$ oxygen via non-rebreather facemask should be the first step in treatment.

- The use of intravenous sedatives should be minimized. Sedation can reduce voluntary efforts to maintain airway patency and risks converting partial occlusion to complete obstruction.

- If direct laryngoscopy is the chosen method of intubation, it should be undertaken with full recognition that visualization of anatomic landmarks and endotracheal tube passage can be challenging due to edema of the pharyngeal wall, epiglottis and vocal cords. The first attempt provides the best opportunity for intubation and should be executed by the most qualified provider.

- It is mandatory to proceed through the airway management protocol with maximal efficiency. Repetition of failed intubation attempts is to be avoided. Delay in the establishment of a surgical airway prolongs the period of hypoxia and can lead to cerebral ischemia.

- If surgical airway access is deemed necessary, cricothyroidotomy provides a rapid means of securing the airway and is the procedure of choice.

\section{CLINICAL SCENARIOS}

\section{Non-Critical Airway Compromise}

For the most common scenario of non-critical airway compromise, the patient is efficiently transported to the operative suite. The surgical team should be assembled and ready with a tracheostomy tray available. Standard monitoring is initiated and the patient is hyper-oxygenated by face mask ventilation. Awake intubation utilizing a fiberoptic (or direct laryngoscopic) technique is attempted if there is imminent danger of losing the airway.

In the case of failed intubation and an obvious spaceoccupying hematoma, the incision is opened under local anesthetic while maintaining bag-mask ventilation. Separation of the subcutaneous tissue is followed by removal of all sutures reapproximating the platysma muscle. Blood clot is evacuated from alongside and posterior to the midline viscera. After decompression of the wound, a second trial of intubation is executed.

If intubation is unsuccessful, a surgical airway will need to be established by cricothyroidotomy. The spine surgeon, anesthesiologist, intensivists, and house staff must be competent in the execution of this procedure. The technique of cricothyroidotomy following anterior spine surgery incorporates extension of the original incision across the midline. The cricothyroid membrane is sharply divided and the opening dilated using a curved hemostat. A small endotracheal or tracheostomy tube $(6-7 \mathrm{~mm}$ diameter $)$ is inserted, and the cuff inflated.

In the presence of a space-occupying lesion, tracheal deviation and submandibular soft tissue swelling can substantially increase the technical difficulty of the surgical procedure. If time allows, recruitment of a specialist with expertise in surgical airway creation is beneficial. General anesthesia is instituted once the endotracheal tube is in place. The surgical team proceeds to formal wound exploration for complete evacuation of the hematoma and control of any ongoing hemorrhage.

\section{Critical Airway Compromise}

In the situation of critical airway compromise, the emergency response system is activated and treatment is initiated at the bedside. The first step is to administer $100 \%$ oxygen via non-rebreather facemask. If adequate bag-mask ventilation can be established, the patient is transported to the operative suite for definitive airway control. If ventilation is inadequate, awake intubation by fiberoptic airway management or direct laryngoscopy is attempted. In the case of successful endotracheal tube placement, transfer to the operating room is arranged for wound exploration.

If the initial intubation attempt fails (or airway management equipment is not immediately available), the surgical wound is opened and blood clot removed. Following wound decompression, another trial of face mask ventilation 
is initiated. If adequate ventilation is not possible and the clinical status allows, an additional attempt at intubation is performed.

If intubation is not rapidly achieved, a surgical airway is established by cricothyroidotomy. Once the endotracheal tube has been secured and ventilation restored, the patient is moved to the operative suite for formal wound exploration and control of any ongoing hemorrhage. Conversion to a tracheostomy can be performed during the same operation or at a later time.

\section{CONCLUSION}

Airway obstruction due to wound hematoma is a rare but potentially lethal complication of anterior cervical spine surgery. This clinical scenario is challenging given the often rapid onset of airway compromise, the limited time to act and the distortion of the upper respiratory tract and ventral neck anatomy. Furthermore, the clinicians of first response are often ill-prepared to deal with this unique type of airway emergency. We report a case of hematoma causing airway compromise following anterior cervical discectomy and fusion in which quick action to evacuate the hematoma and appropriate consultation with otolaryngology to secure the airway led to a successful outcome. There are no Level 1,2 or 3 studies upon which to base guidelines for this challenging clinical scenario. To optimize outcome, specialized knowledge and a systematic approach to management is necessary. Progress through the airway management protocol must be maximally efficient. Delay in securing the airway prolongs the period of hypoxia and can lead to cerebral ischemia and death. As such, all medical personnel responsible for patient care after cervical spine surgery should be aware of this potential complication to respond effectively to this adverse event.

\section{ACKNOWLEDGEMENT}

We would like to acknowledge Michelle L. Daniels, DMD for assistance with illustrations.

\section{CONFLICT OF INTEREST}

Declared none.

\section{REFERENCES}

[1] Tew JM, Mayfield FH. Complications of surgery of the anterior cervical spine. Clin Neurosurg 1976; 23: 424-34.

[2] Bertalanffy H, Eggert HR. Complications of anterior cervical discectomy without fusion in 450 consecutive patients. Acta Neurochir (Wien) 1989; 99: 41-50.

[3] Emery SE, Bohlman HH, Bolesta MJ, Jones PK. Anterior cervical decompression and arthrodesis for the treatment of cervical spondylotic myelopathy: Two to seventeen-year follow-up. J Bone Joint Surg Am 1998; 80A: 941-51.

[4] Marotta N, Landi A, Tarantino R, Mancarella C, Ruggeri A, Delfini R. Five-year outcome of stand-alone fusion using carbon cages in cervical disc arthrosis. Eur Spine J 2011; 20 (Suppl 1): S812 .

[5] Albert T, Balderston R, Northrup B. Surgical approaches to the spine. Philadelphia: WB Saunders 1997; p. 10.

[6] $\mathrm{Yu} \mathrm{NH}$, Jahng TA, Kim CH, Chung CK. Life-threatening late hemorrhage due to superior thyroid artery dissection after anterior cervical discectomy and fusion. Spine (Phila $\mathrm{Pa}$ 1976) 2010; 35(15): E739-42.

[7] Sethi R, Tandon M, Ganjoo P. Neck hematoma causing acute airway and hemodynamic compromise after anterior cervical spine surgery. J Neurosurg Anesthesiol 2008; 20(1): 69-70.

[8] Carr EM, Benjamin E. In vitro study investigating post neck surgery haematoma airway obstruction. J Laryngol Otol 2009; 122: 662-5.

[9] Lee HS, Lee BJ, Kim SW, Cha YW, Choi YS, Park YH, Lee KD. Patterns of post-thyroidectomy hemorrhage. Clin Exp Otorhinolaryngol 2009; 2(2): 72-7.

[10] Gwinnutt CL, Walsh GR, Kumar R. Airway obstruction after anterior cervical spine surgery. J Neurosurg Anesthesiol 1992; 4(3):199-202.

[11] Shakespeare WA, Lanier WL, Perkins WJ, Pasternak JJ. Airway management in patients who develop neck hematomas after carotic endarterectomy. Anesth Analg 2010; 110(2): 588-93.

[12] Sagi HC, Beutler W, Carroll E, Connolly PJ. Airway complications associated with surgery on the anterior cervical spine. Spine 2002 ; 27(9): 949-53.

[13] Kwon B, Yoo JU, Furey CG, Rowbottom J, Emery SE. Risk factors for delayed extubation after single-stage, multi-level anterior cervical decompression and posterior fusion. J Spinal Disord Tech 2006; (19): 389-93.

[14] Terao Y, Matsumoto S, Yamashita K, et al. Increased incidence of emergency airway management after combined anterior-posterior cervical spine surgery. J Neurosurg Anesthesiol 2004; (16): 282-6. 